ECCOMAS

Proceedia
UNCECOMP 2021

$4^{\text {th }}$ ECCOMAS Thematic Conference on Uncertainty Quantification in Computational Sciences and Engineering M. Papadrakakis, V. Papadopoulos, G. Stefanou (eds.) Streamed from Athens, Greece, 28 -30 June 2021

\title{
OPTIMAL SELECTION OF BAYESIAN VIRTUAL SENSORS FOR DAMAGE DETECTION UNDER VARIABLE ENVIRONMENTAL CONDITIONS
}

\author{
Jyrki Kullaa \\ Department of Automotive and Mechanical Engineering \\ Metropolia University of Applied Sciences \\ P.O. Box 4071, 00079 Metropolia, Finland \\ e-mail: jyrki.kullaa@metropolia.fi
}

\begin{abstract}
Measuring structural vibrations with a large sensor network results in lots of data in structural health monitoring applications. A large number of sensors is advantageous for damage detection and localization. By storing only a few selected Bayesian virtual sensors it is possible to decrease the amount of data and reconstruct the discarded sensor data even with higher accuracy than the original measurements. A method is proposed, in which the stored and reconstructed data are used for damage detection and localization in the time domain. A numerical experiment was performed with a structure having a large number of sensors. The excitation and environmental conditions were variable and unknown. An optimal sensor placement algorithm was applied individually to each measurement to select the appropriate virtual sensors for storage. Less than ten percent of the data were stored, and the signals of all the reconstructed sensors were still more accurate than the actual measurements. The stored and reconstructed data outperformed the actual measurement data in damage detection and localization. Surprisingly, damage detection was also more successful with the stored and reconstructed data than with the full set of virtual sensors.
\end{abstract}

Keywords: Data Compression, Virtual Sensing, Damage Detection, Optimal Sensor Placement, Environmental Effects, Sensor Network. 


\section{INTRODUCTION}

Structural health monitoring (SHM) produces lots of data. Vibrations of structures are measured frequently using a sensor network with tens or hundreds of sensors. There is an increasing interest to develop dense sensor networks for SHM applications, for example sensing skins [1]. With an increasing number of sensors, damage detection and localization become more reliable, but at the expense of higher data management costs. Historical data must be stored for unsupervised learning to train the model of the undamaged structure under different environmental or operational conditions. Storing such a large amount of data during years of monitoring may be difficult and costly. Therefore, data reduction would be necessary. Data can be reduced using multivariate statistical techniques for dimensionality reduction. The most common dimensionality reduction method is principal component analysis (PCA) [2], which is a linear method that maximizes the variance in the data by projecting the data onto directions, principal components (PC) that account for the largest variability. If only a few PCs are retained, some loss of data results. PCA has been applied e.g. to image compression [3].

Another method for data reduction is to extract selected features from the time records and store these features only. Features are dynamic characteristics of the structure, which are expected to be sensitive to damage. Such features are for example natural frequencies and mode shapes, which can be extracted from the measurement data using system identification techniques [4]. Significant data compression occurs, because a single feature vector (one data point) only results from each measurement. The dimensionality of the feature vector is, however, often higher than the number of sensors. This may result in the curse of dimensionality in statistical data analysis. In addition, the time histories will be lost and cannot be recovered in case new potential features are later considered. Therefore, it may be necessary to save everything resulting in terabytes of data every day [5].

If only a limited number of sensor signals are permanently stored, the sensors must be selected according to some criterion. This selection is associated with optimal sensor placement (OSP), which has been studied for different applications including vibration control, experimental modal analysis, model updating, fault detection, and impact identification [6]. Although the objective in these applications is to place a limited amount of physical sensors in optimal positions, the same approach can be used to select a subset of measurements for storage. The selection criterion is related to the accuracy of the reconstructed signals.

Some review papers and comparisons of different optimal sensor placement algorithms exist [7-10]. They present the most commonly applied algorithms and criteria. Sensor placement is a discrete optimization problem, for which genetic algorithms have been proposed [11-13]. Alternatively, a computationally efficient and widely used algorithm is to start with a large set of candidate sensor locations and remove one sensor in each round based on the selected cost function until the required criterion is violated. This backward sequential sensor placement (BSSP) algorithm has been used in many studies [14-16]. Another iterative method is to add one sensor in turn to the sensor network until the stopping criterion is met. The algorithm is called forward sequential sensor placement (FSSP) algorithm $[11,16]$. BSSP is used in this study.

If the number of sensors in the network is larger than the number of active modes, the sensor network is redundant. The redundancy can be utilized to estimate the quantity of interest using virtual sensing techniques. Virtual sensing (VS) can be either model-based (analytical) or data-driven (empirical) [17]. In analytical virtual sensing, in addition to measurement data, a numerical model of the structure is needed, for example a finite element model. Empirical virtual sensing is based on training data from a redundant full sensor network. It can be used, 
for example, to replace a temporarily installed or failed sensor [18]. Empirical virtual sensing has also been used in structural dynamics for damage detection [19] or sensor fault detection [20].

In this paper, virtual sensors are developed for data compression and accurate reconstruction in a large sensor network. The objective is to detect and localize damage using the stored and reconstructed virtual sensor data.

With empirical Bayesian virtual sensing, the resulted accuracy of the virtual sensors is higher than that of the physical hardware [21]. A limited number of virtual sensors are stored, from which the discarded signals can be reconstructed. Optimal sensor placement is studied for the most accurate reconstruction of the excluded data. The cost function in the sensor placement optimization is related to the reconstruction error, which must be minimized.

Damage detection is based on changes in the dynamic characteristics of the structure. Records of structural motion, for example acceleration, are measured simultaneously at selected degrees-of-freedom. First, a training data set is acquired from the undamaged structure under different environmental or operational conditions. These data are used to build a statistical data model of the undamaged structure. Next, the structure is being monitored with repeated measurements in order to have an early warning of structural failure. The new test data are compared to the training data using novelty detection techniques, and a statistically significant change in the dynamic characteristics is an indication of damage. Particular attention is needed to take variable environmental or operational conditions into account, because they can have a considerable influence on the very same dynamic characteristics. Several techniques have been proposed to eliminate the environmental or operational influences on the data, even without measuring the underlying quantities, see e.g. $[19,22]$ and the references therein. Damage localization can also be attempted if the changes in the data can be assigned to a particular sensor.

In this paper, damage detection and localization are performed in the time domain. The data are the stored and reconstructed virtual sensors. Statistically significant differences between the training and test data are assumed to reveal damage. The largest difference is assumed to localize damage close to the corresponding sensor.

The paper is organized as follows. Virtual sensing using Bayesian estimation is outlined in Section 2. Optimal sensor placement for virtual sensing is also discussed. An algorithm for damage detection and localization follows in Section 3. In Section 4, the proposed method is studied with numerical simulations of ambient vibration measurements. Concluding remarks are given in Section 5.

\section{VIRTUAL SENSING AND OPTIMAL SENSOR PLACEMENT}

The objective is to store only a small percentage of the dense sensor network data so that the full data can be accurately reconstructed for damage detection. One possible data compression technique is Bayesian virtual sensing, which is applied to the whole sensor network, and a selected set of the resulting virtual sensors are only stored. The data from the discarded sensors can be reconstructed using the stored signals.

\subsection{Empirical Bayesian virtual sensing}

Empirical virtual sensing is based on available current or historical measurements. Consider a sensor network measuring $p$ simultaneously sampled response variables $\mathbf{y}=\mathbf{y}(t)$ at time $t$. Each measurement $\mathbf{y}$ includes measurement error $\mathbf{w}=\mathbf{w}(t)$ :

$$
\mathbf{y}=\mathbf{x}+\mathbf{w}
$$


where $\mathbf{x}=\mathbf{x}(t)$ are the exact values of the measured degrees of freedom. Equation 1 can be written in the following form at time $t$ [23].

$$
\left\{\begin{array}{l}
\mathbf{x} \\
\mathbf{y}
\end{array}\right\}=\left[\begin{array}{ll}
\mathbf{I} & \mathbf{0} \\
\mathbf{I} & \mathbf{I}
\end{array}\right]\left\{\begin{array}{l}
\mathbf{x} \\
\mathbf{w}
\end{array}\right\}
$$

For simplicity but without loss of generality, assume zero-mean variables $\mathbf{x}$ and $\mathbf{y}$. The partitioned covariance matrix is

$$
\begin{aligned}
& {\left[\begin{array}{ll}
\boldsymbol{\Sigma}_{x x} & \boldsymbol{\Sigma}_{x y} \\
\boldsymbol{\Sigma}_{y x} & \boldsymbol{\Sigma}_{y y}
\end{array}\right]=\mathrm{E}\left(\left\{\begin{array}{l}
\mathbf{x} \\
\mathbf{y}
\end{array}\right\}\left[\begin{array}{ll}
\mathbf{x}^{T} & \mathbf{y}^{T}
\end{array}\right]=\left[\begin{array}{ll}
\mathbf{I} & \mathbf{0} \\
\mathbf{I} & \mathbf{I}
\end{array}\right] \mathrm{E}\left(\begin{array}{l}
\mathbf{x} \\
\mathbf{w}
\end{array}\right\}\left[\begin{array}{ll}
\mathbf{x}^{T} & \mathbf{w}^{T}
\end{array}\right]\left[\begin{array}{ll}
\mathbf{I} & \mathbf{0} \\
\mathbf{I} & \mathbf{I}
\end{array}\right]^{T}\right.} \\
& =\left[\begin{array}{ll}
\mathbf{I} & \mathbf{0} \\
\mathbf{I} & \mathbf{I}
\end{array}\right]\left[\begin{array}{cc}
\boldsymbol{\Sigma}_{x x} & \mathbf{0} \\
\mathbf{0} & \boldsymbol{\Sigma}_{w w}
\end{array}\right]\left[\begin{array}{ll}
\mathbf{I} & \mathbf{I} \\
\mathbf{0} & \mathbf{I}
\end{array}\right]=\left[\begin{array}{cc}
\boldsymbol{\Sigma}_{x x} & \boldsymbol{\Sigma}_{x x} \\
\boldsymbol{\Sigma}_{x x} & \boldsymbol{\Sigma}_{x x}+\boldsymbol{\Sigma}_{w w}
\end{array}\right]
\end{aligned}
$$

where $\mathrm{E}(\cdot)$ denotes the expectation operator and the measurement error $\mathbf{w}$ is assumed to be zero mean Gaussian, independent of $\mathbf{x}$, with a covariance matrix $\boldsymbol{\Sigma}_{w w}$.

A linear minimum mean square error (MMSE) estimate for $\mathbf{x} \mid \mathbf{y}$ (x given $\mathbf{y}$ ) is obtained by minimizing the mean-square error (MSE) [20, 23]. The expected value, or the conditional mean, of the predicted variable is:

$$
\hat{\mathbf{x}}=\mathrm{E}(\mathbf{x} \mid \mathbf{y})=\boldsymbol{\Sigma}_{x x}\left(\boldsymbol{\Sigma}_{x x}+\boldsymbol{\Sigma}_{w w}\right)^{-1} \mathbf{y}=\boldsymbol{\Sigma}_{x x} \boldsymbol{\Sigma}_{y y}^{-1} \mathbf{y}
$$

and the estimation error is

$$
\boldsymbol{\Sigma}_{\text {post }}=\operatorname{cov}(\mathbf{x} \mid \mathbf{y})=\boldsymbol{\Sigma}_{x x}-\boldsymbol{\Sigma}_{x x}\left(\boldsymbol{\Sigma}_{x x}+\boldsymbol{\Sigma}_{w w}\right)^{-1} \boldsymbol{\Sigma}_{x x}=\boldsymbol{\Sigma}_{x x}-\boldsymbol{\Sigma}_{x x} \boldsymbol{\Sigma}_{y y}^{-1} \boldsymbol{\Sigma}_{x x}
$$

The covariance matrix $\boldsymbol{\Sigma}_{x x}$ is not known, but $\boldsymbol{\Sigma}_{y y}$ can be estimated from the measurement data. If the noise covariance matrix can be approximated, then an estimate for $\boldsymbol{\Sigma}_{x x}=\boldsymbol{\Sigma}_{y y}-\boldsymbol{\Sigma}_{w w}$ can be computed. In this study, measurement errors are assumed uncorrelated between sensors resulting in a diagonal noise covariance matrix. In addition, because $\boldsymbol{\Sigma}_{x x}$ must be positive definite, an upper bound of the noise level in each sensor can be obtained [21].

\subsection{Data compression}

After a single measurement, the data from all sensors are available. If only a subset of the signals is stored, a lot of disc storage space can be saved. Let us assume that only channels $v$ are stored. The stored signals can be either the actual measurements $\mathbf{y}_{v}$ or virtual sensors $\hat{\mathbf{x}}_{v}$. It was proved [24] that the stored Bayesian virtual sensors $\hat{\mathbf{x}}_{v}$ outperform the corresponding raw measurements $\mathbf{y}_{v}$ resulting in a smaller reconstruction error and should be preferred. Therefore, the stored data in this paper are from the virtual sensors.

Let us assume that channels $v$ of the virtual sensors are stored, while the remaining channels $u$ must be reconstructed. Storing the virtual sensors $\hat{\mathbf{x}}_{v}$, the conditional mean $\mathrm{E}\left(\mathbf{x}_{u} \mid \hat{\mathbf{x}}_{v}\right)$ and covariance matrix $\operatorname{cov}\left(\mathbf{x}_{u} \mid \hat{\mathbf{x}}_{v}\right)$ must be derived. The Bayesian virtual sensors are not exact, but follow the error model

$$
\mathbf{x}=\hat{\mathbf{x}}+\mathbf{e}
$$

where $\mathbf{e}$ is the posterior error (5) having a zero mean. Thus,

$$
\mathrm{E}\left(\mathbf{x}_{u} \mid \hat{\mathbf{x}}_{v}\right)=\mathrm{E}\left(\hat{\mathbf{x}}_{u} \mid \hat{\mathbf{x}}_{v}\right)=\boldsymbol{\Sigma}_{\hat{x}, u v} \boldsymbol{\Sigma}_{\hat{x}, v v}^{-1} \hat{\mathbf{x}}_{v}=\mathbf{A} \hat{\mathbf{x}}_{v}
$$


where $\mathbf{A}=\boldsymbol{\Sigma}_{\hat{x}, u v} \boldsymbol{\Sigma}_{\hat{x}, v v}^{-1}$ is the coefficient matrix that has to be stored together with the stored signals $\hat{\mathbf{x}}_{v}$. According to MMSE, the two terms in the right hand side of (6) are orthogonal [25]. Therefore, the covariances are related as

$$
\begin{aligned}
& \operatorname{cov}\left(\mathbf{x}_{u} \mid \hat{\mathbf{x}}_{v}\right)=\operatorname{cov}\left(\hat{\mathbf{x}}_{u} \mid \hat{\mathbf{x}}_{v}\right)+\boldsymbol{\Sigma}_{\text {post }, u u} \\
& =\boldsymbol{\Sigma}_{\hat{x}, u u}-\boldsymbol{\Sigma}_{\hat{x}, u v} \boldsymbol{\Sigma}_{\hat{x}, v v}^{-1} \boldsymbol{\Sigma}_{\hat{x}, v u}+\boldsymbol{\Sigma}_{\text {post }, u u}
\end{aligned}
$$

which means that the error of the reconstructed sensor is larger than that in the Bayesian virtual sensor (5). This difference in accuracy is studied in Section 4.1.

\subsection{Optimal sensor placement}

The proposed optimal sensor placement algorithm is an iterative procedure starting with an initial large sensor network including all measured degrees-of-freedom (DOF). Each sensor in turn is removed with replacement, and the error variances of all reconstructed signals are computed, which are the diagonal terms of the error covariance matrix (8). The cost function for these reduced sensor networks is evaluated. The minimum cost is found and the reduced sensor network corresponding to this minimum becomes the new candidate set for the next round. In other words, the removed sensor corresponding to this minimum cost is permanently discarded. The process is repeated until the desired number of sensors or the allowed error limit is reached. Finally, the data from the remaining sensors are stored together with matrix A in (7) for reconstruction of the discarded sensors.

\section{DAMAGE DETECTION AND LOCALIZATION}

Damage detection is applied to the reconstructed data in the time domain. First, the mean vector and the covariance matrix are estimated using training data from the undamaged structure under different environmental or operational conditions. Whitening transformation is applied to the training data [26]. This transformation is then fixed and applied to the test data. The residual errors between the model and actual data are computed and subjected to principal component analysis (PCA). Retaining the first principal component scores of the residuals, the data dimensionality is decreased to one. An extreme value statistics control chart is then designed for the first PC scores of the residuals with appropriate control limits and subgroup size $[19,27,28]$. In this paper, the probability of false alarms equal to 0.001 has been used.

Damage location is assumed to correspond to the direction of the first principal component of the residuals. The largest projection of the first PC on the sensor coordinates reveals the sensor closest to damage.

It is essential to model the data of each measurement independently for compression and reconstruction so that the environmental or operational or damage effects are retained during this first phase. Elimination of the environmental or operational influences is performed only in the second phase in which several measurements are pooled to build a data model of the undamaged structure under different environmental or operational conditions. Novelty detection is then applied to the test data using the data model of the second phase.

\section{NUMERICAL EXPERIMENT}

A numerical experiment was performed with a finite element (FE) model of a steel frame having a height of $4.0 \mathrm{~m}$ and a width of $3.0 \mathrm{~m}$ (Figure 1). The columns were fixed at the bottom. The frame was also supported with a horizontal spring at the elevation of $2.75 \mathrm{~m}$ with a spring constant of $k=2.0 \mathrm{MN} / \mathrm{m}$. The frame was modelled with simple beam elements having 
a hollow square cross section of $100 \mathrm{~mm} \times 100 \mathrm{~mm} \times 5 \mathrm{~mm}$. The FE model consisted of 176 beam elements $62.5 \mathrm{~mm}$ in length and a single spring element.

Three horizontal random excitations were applied to the right column at elevations of $4 \mathrm{~m}$, $3 \mathrm{~m}$, and $2 \mathrm{~m}$, respectively (Figure 1). The loads were mutually independent having random standard deviations between $100 \mathrm{~N}$ and $900 \mathrm{~N}$. Periodic pseudorandom excitations in the frequency range between 0 and $53.33 \mathrm{~Hz}$ with random amplitudes and phases were generated [3]. All analyses had different loading functions. The first seven modes were used in the simulation. Modal damping was assumed with damping ratios of $\zeta_{1-2}=0.01, \zeta_{3}=0.015$, and $\zeta_{4-7}=$ 0.02 .

Steady state analysis was performed in the frequency domain using modal superposition. Lateral accelerations at 59 points (every third node) were recorded. The sampling frequency was $250 \mathrm{~Hz}$ and the measurement period was $32.77 \mathrm{~s}$. Each sensor thus produced $8192 \mathrm{sam}-$ ples. Mutually independent Gaussian random noise with equal standard deviations was added to each sensor. The average SNR was $30 \mathrm{~dB}$. For validation and comparison, exact transverse accelerations were also recorded. The standard deviation of the noise was assumed to be known.

A relatively complex but also quite realistic environmental model was applied. The temperature of the left upper corner, $T_{65}$ varied randomly between $-25^{\circ} \mathrm{C}$ and $+40^{\circ} \mathrm{C}$. The subscript 65 indicates the node number. The temperature of the other end points varied randomly: $T_{113}=T_{65} \pm 5^{\circ} \mathrm{C}$ (upper right corner); $T_{1}=T_{65} \pm 3^{\circ} \mathrm{C}$ (bottom left support); and $T_{177}=T_{113} \pm$ $3^{\circ} \mathrm{C}$ (bottom right support). Temperature variation between the aforementioned points was linear except that Gaussian random error with a standard deviation of $0.2^{\circ} \mathrm{C}$ was added to each element. The relationship between temperature and the Young's modulus $E$ was stepwise linear as shown in Fig. 2a. Sample distributions of the Young's modulus in the elements are plotted in Fig. 2b. Within each short measurement, the distribution did not change.

Due to the temperature effect, the natural frequencies varied between measurements. Fig. 3 shows the seven lowest natural frequencies of the structure in all measurements. The data points on the right hand side of the vertical line are from the damaged structure. It is difficult to detect damage visually from the frequency changes due to the strong environmental effect.

Damage was removal of material inside a beam element due to corrosion. The damaged element was located at the bottom of the left leg (element 1). Five different damage levels were considered with the wall thicknesses of $4.5,4.0,3.5,3.0$, and $2.5 \mathrm{~mm}$. Notice that as the material was removed, both the stiffness and mass were decreased.

The first 100 measurements were taken from the undamaged structure and each damage level was monitored with six measurements under different and unknown environmental conditions. Training data were the first 70 measurements. The extreme value statistics (EVS) control charts were designed using the same training data. 


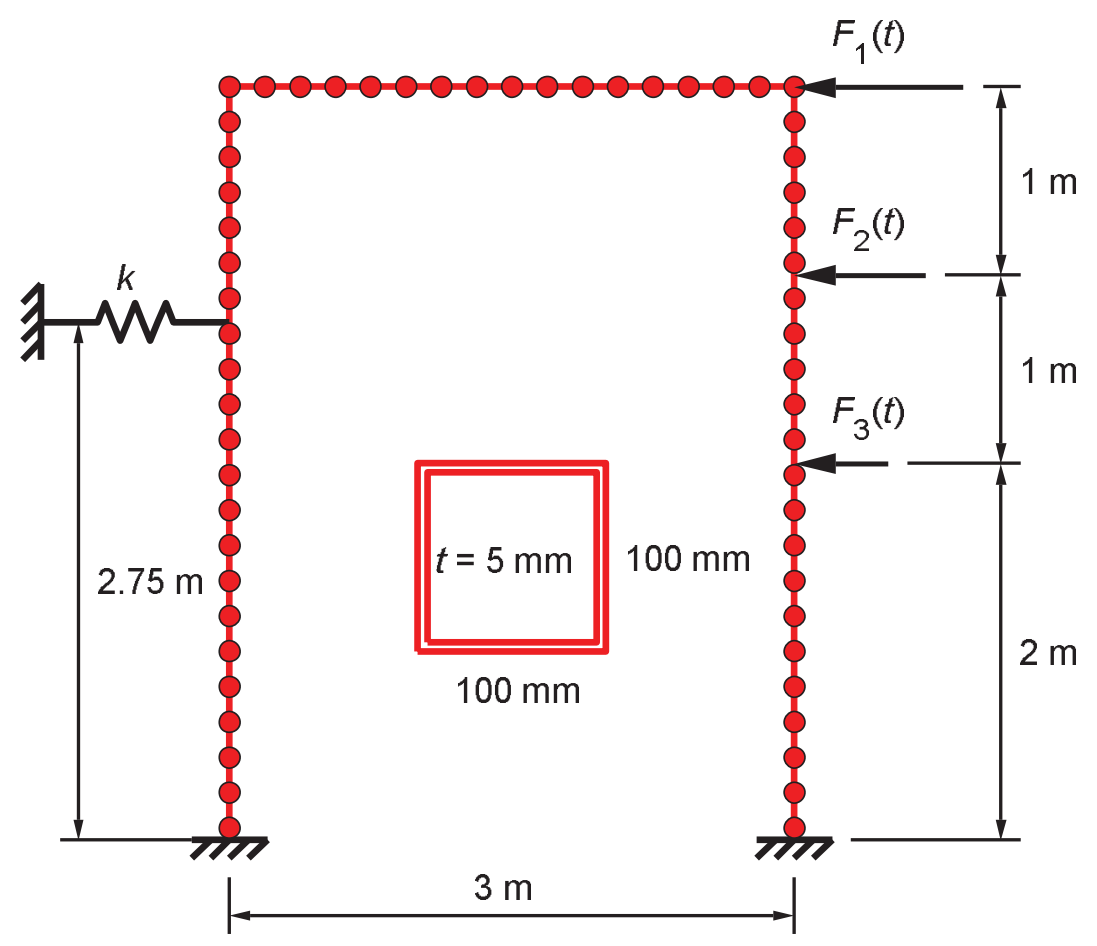

Figure 1: Frame structure with 59 accelerometers.

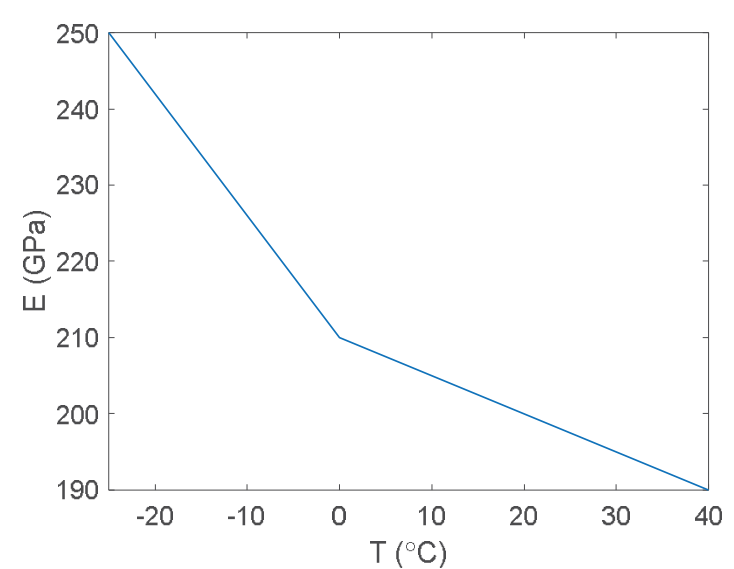

(a)

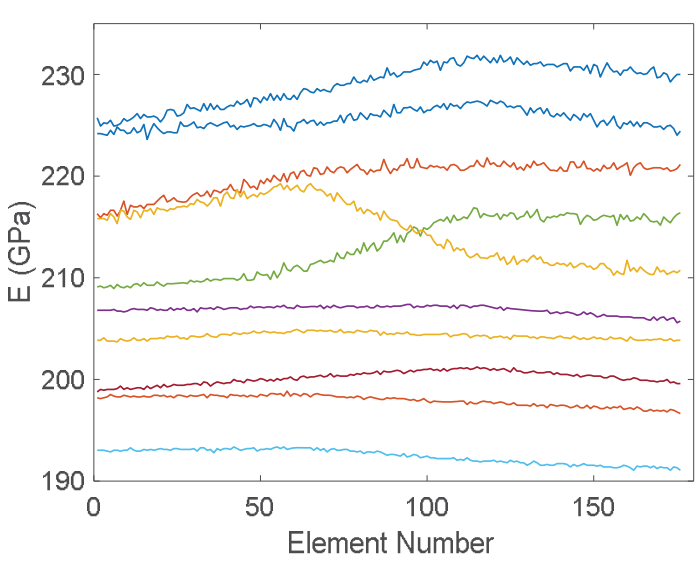

(b)

Figure 2: (a) Young's modulus versus temperature. (b) Sample distributions of the Young's modulus. 


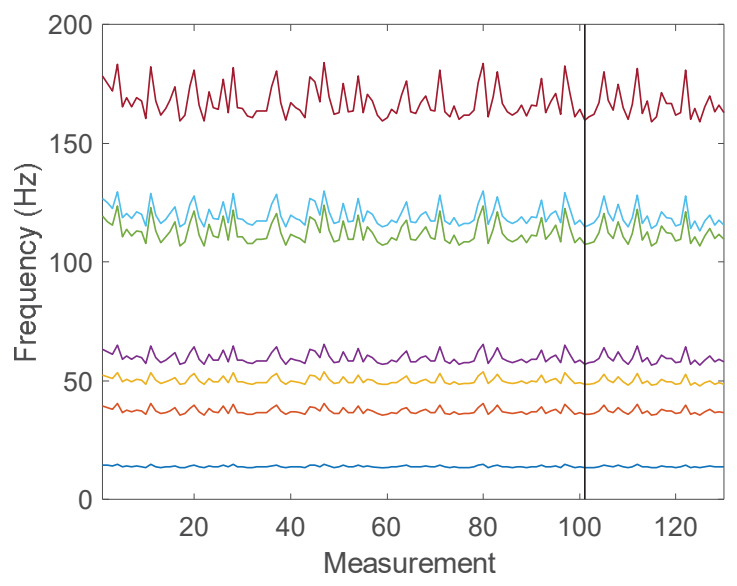

Figure 3: Variation of the seven lowest natural frequencies due to temperature and damage. Frequencies on the right of the vertical line are from the damaged structure.

\subsection{Bayesian virtual sensing and sensor selection}

Bayesian virtual sensing was applied individually to each measurement resulting in noise reduction. A detail of the measured and estimated accelerations of sensor 2 in measurement 1 is plotted in Figure 4. Also the exact values are shown. It can be seen that Bayesian virtual sensor was more accurate than the physical sensor.

Next, a subset of the Bayesian virtual sensors was selected separately for each measurement by applying the backward sequential sensor placement (BSSP) algorithm. The requirement was that noise had to be decreased at least $50 \%$ in all sensors. In other words, the standard deviation of the error in each reconstructed virtual sensor had to be less or equal to half of that of the corresponding measurement error. The cost function was the maximum difference between the current and allowed reconstruction errors in any sensor in the network. The reduced network having the minimum cost was selected for the next round. In other words, the aim was to maximize the minimum distance from the error limit. Sensor removal continued until the accuracy requirement was violated. The required number of virtual sensors was five for most measurements.

Once a single sensor was permanently removed, the mean error of the whole sensor network (stored and reconstructed virtual sensors) was evaluated. The mean error as a function of the number of stored sensors is plotted in Figure 5 for measurement 1. It can be seen that storing only five virtual sensors instead of all 59 virtual sensors did not significantly increase the average noise level. If the number of stored sensors were further decreased below five, the reconstruction error would have considerably increased.

The standard deviations of the errors (measurement error, Bayesian virtual sensor error, and reconstruction error) in all sensors are plotted in Figure 6 for measurement 1 . It can be seen that the reconstruction error was only slightly larger than that in the Bayesian virtual sensors. Sensors, for which the two errors were equal, corresponded to the stored signals, which were not reconstructed. The measurement error is also shown. The reconstruction errors were clearly smaller than requested.

The reconstruction errors in all measurements are plotted in Figure 7 for each sensor. The variability between measurements was quite small satisfying the accuracy requirement.

A histogram of the selected sensors for storage in all measurements is shown in Figure 8 left. The most often selected sensors were located in six different regions of the structure. The placement of the stored sensors in measurement 1 is plotted in Figure 8 right. Notice that no sensors were selected close to damage location (sensor 1). 
The data compression ratio was computed as follows. If all data were stored, the number of floating point numbers in each measurement was $59 \times 8192=483,328$ numbers. Storing five virtual sensor signals and the coefficient matrix A (7) of size $54 \times 5$ resulted in 41,230 numbers. Consequently, only $8.5 \%$ of the total data had to be stored.

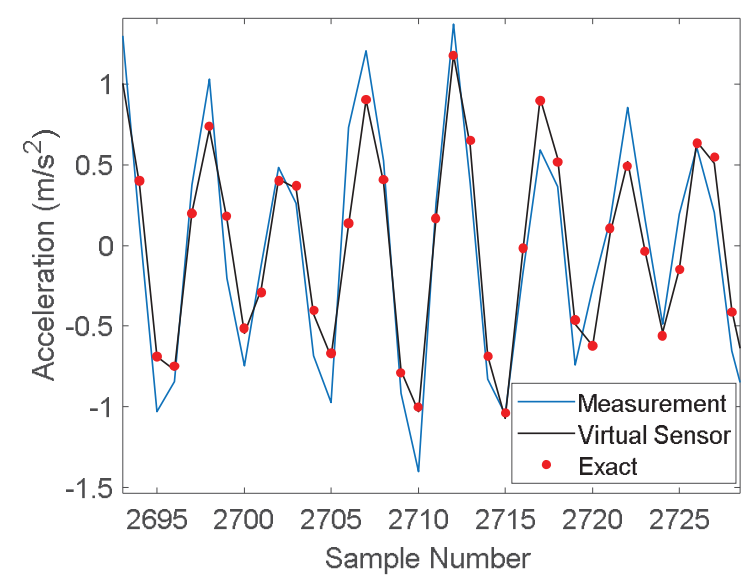

Figure 4: Detail of time history of accelerometer 2 in measurement 1.

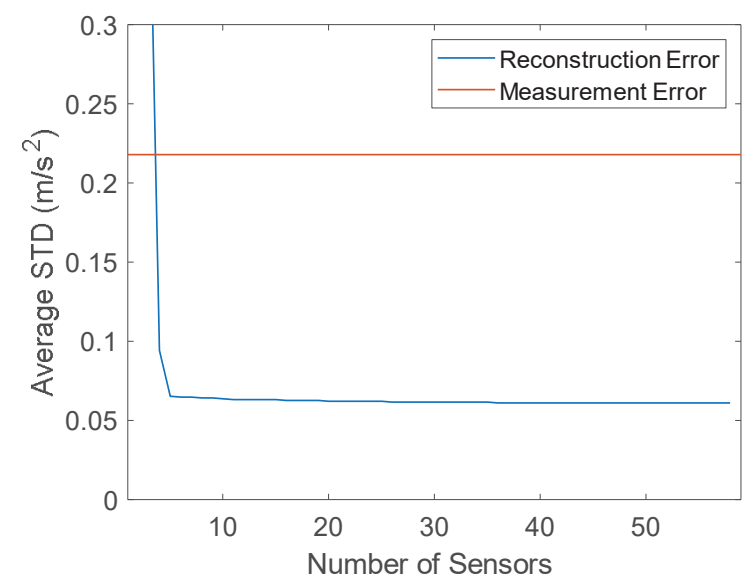

Figure 5: Mean reconstruction error as a function of the number of stored signals in measurement 1 . The red horizontal line is the measurement error.

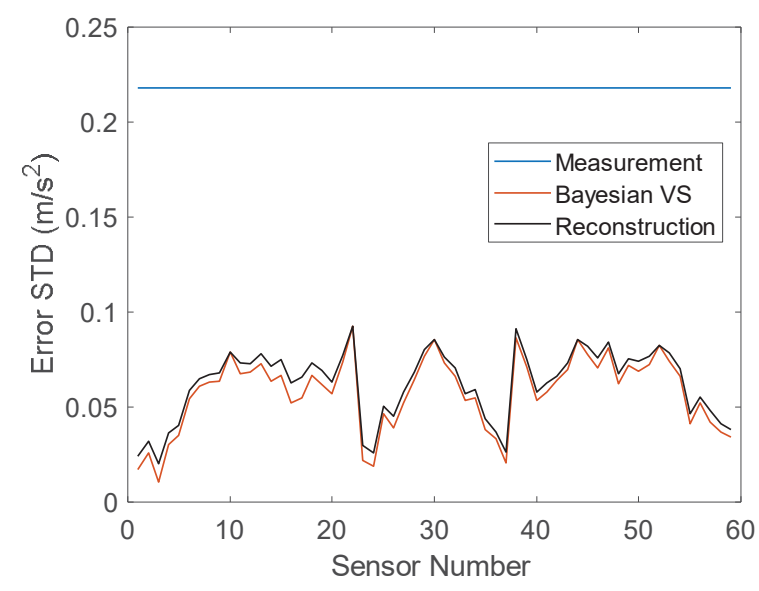

Figure 6: Measurement error, virtual sensor error and the reconstruction error in all sensors in measurement 1. 


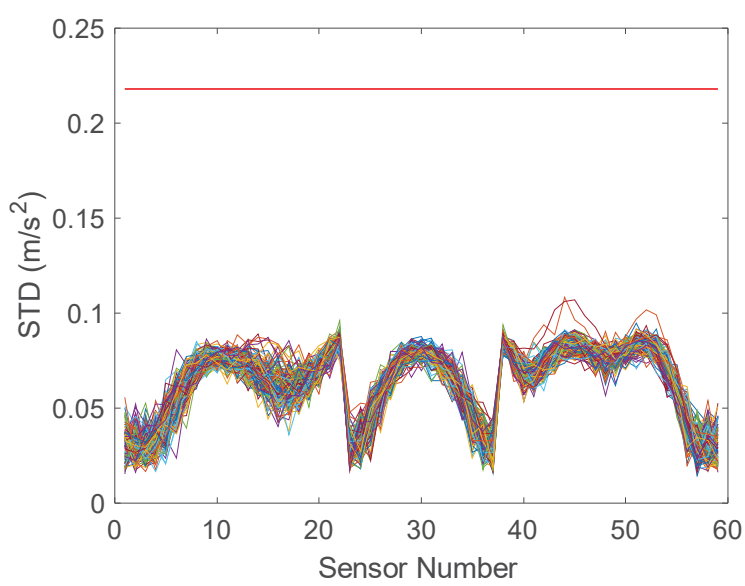

Figure 7: Measurement error (red horizontal line) and the reconstruction error in all sensors in each measurement.
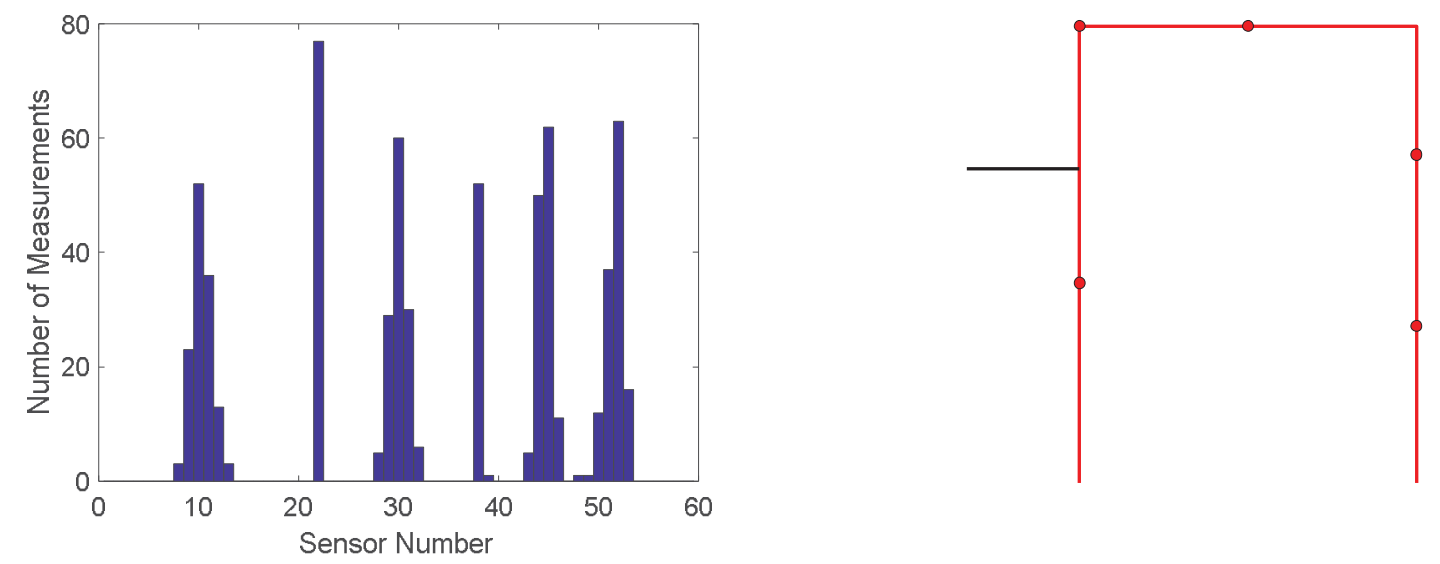

Figure 8: Left: Histogram of selected virtual sensors for storage in all measurements. Right: Selected sensors for storage in measurement 1 : sensors $10,22,30,44$, and 52.

\subsection{Damage detection and localization}

Damage detection was studied using four different data: actual measurements, all virtual sensors, stored and reconstructed signals, and stored signals only. EVS control charts were designed with a subgroup size of 1000 and are plotted in Figure 9. The data points to the left of the blue vertical line correspond to the training data, while the black vertical lines indicate the five damage levels. Only the two largest damage levels were detected using the actual measurement data (Figure 9a). All damage cases were detected using the stored and reconstructed data (Figure 9b) or all virtual sensors (Figure 9c). There is a slight difference between the two control charts showing that the detection performance increased due to compression. This was quite a surprise, because the noise level in the reconstructed data was slightly larger than in the Bayesian virtual sensor data. The reason for this behavior is not known and it is questionable if this result can be generalized.

It may be argued that due to redundancy, only the selected virtual sensors would be enough for damage detection. This argument was tested by selecting the same five virtual sensors from each measurement and designing an EVS control chart for these data (Figure 9d). No damage was detected. Due to different environmental conditions between measurements, more than five signals would have been needed to remove the environmental influences. 
Damage localization was done by plotting the squared projection of the first principal component on each sensor (Figure 10). Using the actual measurement data, damage was localized to sensor 6 , and using the stored and reconstructed virtual sensors, damage was localized to sensor 4. Notice that sensor 4 was not included in the stored sensors but was reconstructed. The correct position was closest to sensor 1 . Neither analysis pointed to the correct sensor, but in either case, the suggested damage location was in the vicinity of the actual damage. The localization accuracy was slightly higher when the virtual sensors were used. The SNR in sensor 1 was very small, which probably resulted in the inaccuracy in damage localization. In many structures, damage may be located close to the fixed support, where the stresses are large but the vibration amplitude is very small resulting in a small SNR. Therefore, strain measurements at these locations could be considered.

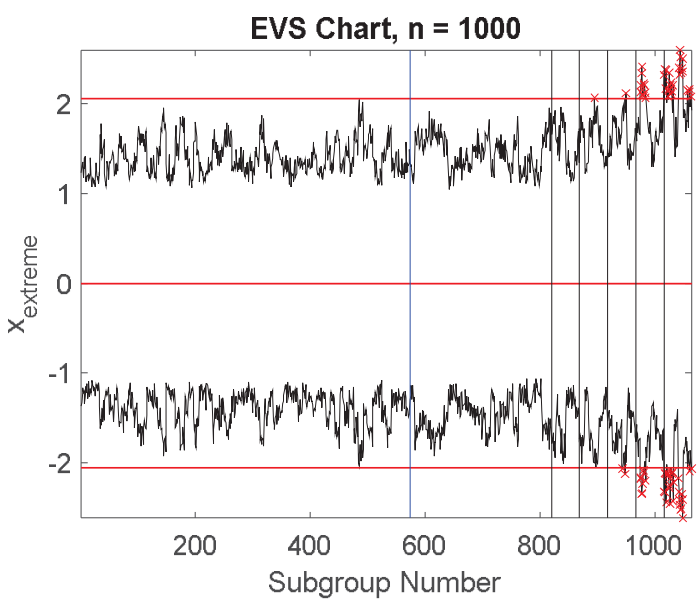

(a)

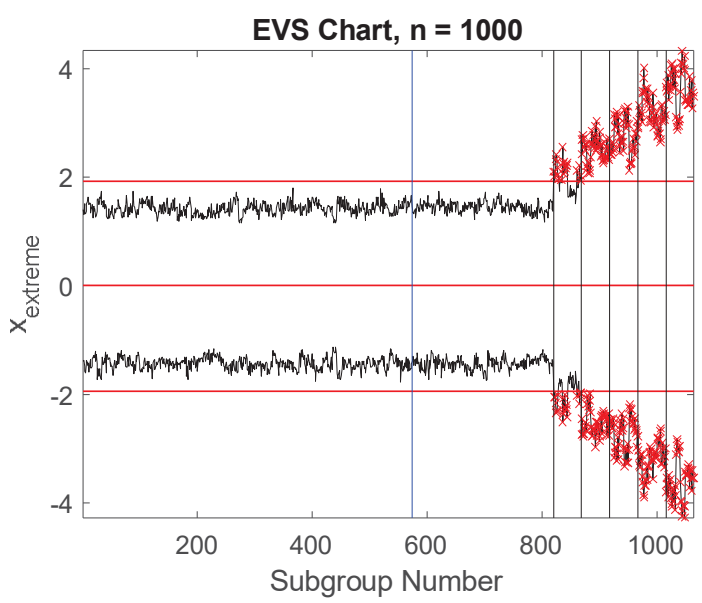

(c)

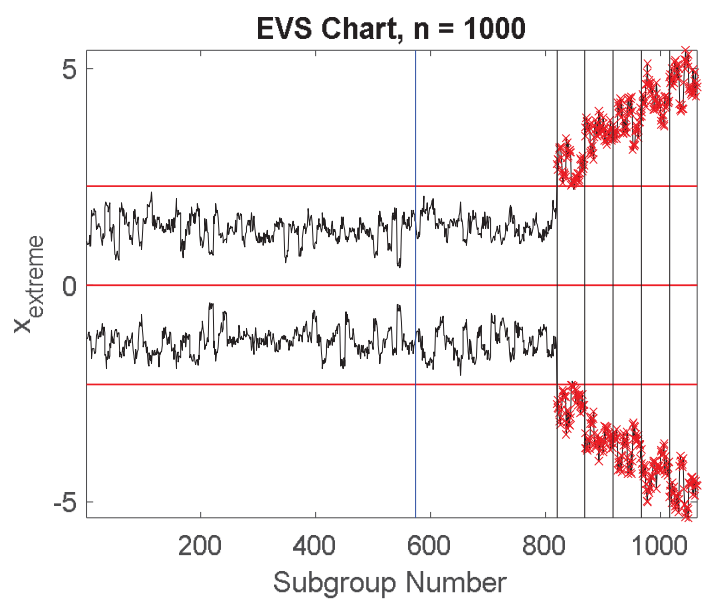

(b)

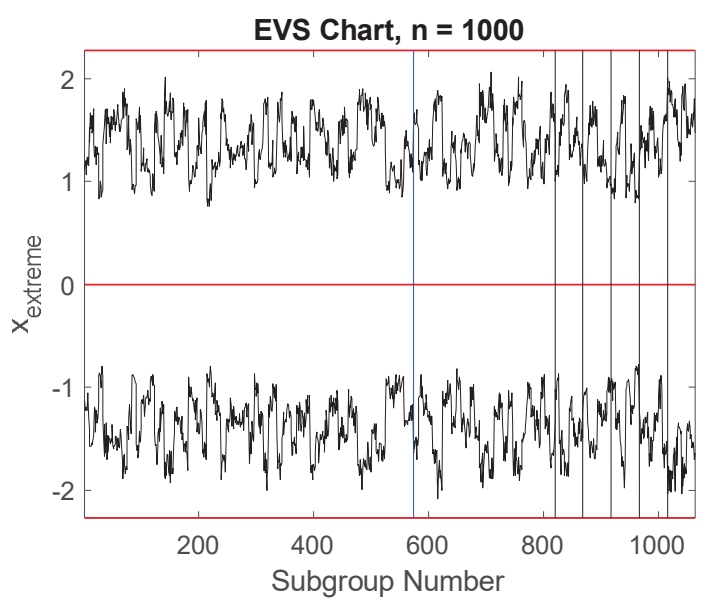

(d)

Figure 9: Damage detection. (a) All physical sensors, (b) stored and reconstructed sensors, (c) all Bayesian virtual sensors, and (d) stored 5 virtual sensors. The vertical lines correspond to the end of training data (blue) and the five damage levels (black). 

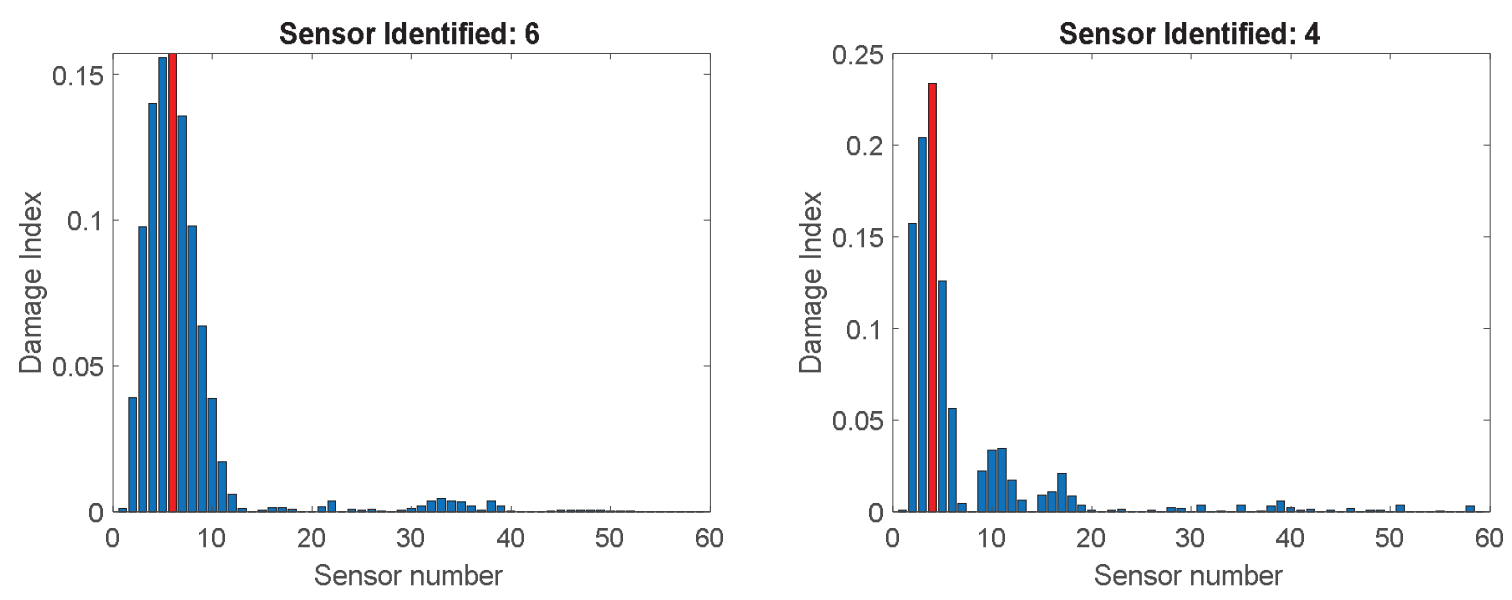

Figure 10: Damage localization. (a) All physical sensors, and (b) stored and reconstructed virtual sensors. The correct damage position was closest to sensor 1 .

\section{CONCLUSION}

A data compression technique for storing and reconstructing simultaneously measured vibration signals in a dense sensor network was proposed. The stored and reconstructed data were used to detect and localize damage.

The first step was to reduce measurement error by applying Bayesian virtual sensing. The virtual sensors, being more accurate than the physical sensors, replaced the actual measurements in the subsequent steps.

Data compression and reconstruction was made individually for each measurement, because the dynamic characteristics of the structure could vary between measurements due to environmental or operational variability or damage. On the other hand, a full set of training data from several measurements under different environmental or operational conditions was used to build a covariance model of the undamaged structure. This model was applied to novelty detection using whitening transformation and principal component analysis. The largest discrepancy between the model and actual data was assumed to reveal the sensor closest to the damage location.

The main results are: (1) Only around $8.5 \%$ of the total amount of data had to be stored in the studied example. (2) The stored and reconstructed virtual sensor data were more accurate than the actual measurements. (3) Damage detection and localization were more reliable with the stored and reconstructed virtual sensors than with the actual measurements. (4) Damage was not localized exactly to the correct sensor but in the close neighborhood. (5) The accuracy of the reconstructed virtual sensors was only slightly smaller than that of the Bayesian virtual sensors. (6) The reconstruction errors were not the same even if the measurement errors were equal. (7) Damage localization to a reconstructed virtual sensor was possible. (8) Damage detection performance was slightly higher using the stored and reconstructed data than all virtual sensors, but generalization of this result remained questionable and needs further investigation. Experimental results are also needed to validate the proposed technique.

\section{ACKNOWLEDGEMENTS}

This research was supported by Metropolia University of Applied Sciences. 


\section{REFERENCES}

[1] M. Sadoughi, A. Downey, J. Yan, C. Hu, S. Laflamme, Reconstruction of unidirectional strain maps via iterative signal fusion for mesoscale structures monitored by a sensing skin. Mechanical Systems and Signal Processing, 112, 401-416, 2018.

[2] S. Sharma, Applied multivariate techniques. Wiley, 1996.

[3] A. Brandt, Noise and vibration analysis: Signal analysis and experimental procedures. Wiley, 2011.

[4] R. Brincker, C. Ventura, Introduction to operational modal analysis. Wiley, 2015.

[5] P. Cawley, Structural health monitoring: closing the gap between research and industrial deployment. Structural Health Monitoring, 17, 1225-1244, 2018.

[6] V. Mallardo, M. Aliabadi, Optimal sensor placement for structural, damage and impact identification: A review, Structural Durability \& Health Monitoring, 9, 287-323, 2013.

[7] T-H. Yi, H-N. Li, Methodology developments in sensor placement for health monitoring of civil infrastructures, International Journal of Distributed Sensor Networks, 15501329, 2012.

[8] A. Krause, C. Guestrin, A. Gupta, J. Kleinberg, Near-optimal sensor placements: maximizing information while minimizing communication cost, Proceedings of the $5^{\text {th }}$ International Conference on Information Processing in Sensor Networks (IPSN '06), New York, NY, USA, 2006, 2-10.

[9] M. Meo, G. Zumpano, On the optimal sensor placement techniques for a bridge structure, Engineering Structures, 27, 1488-1497, 2005.

[10] C. Leyder, E. Chatzi, A. Frangi, G. Lombaert, Comparison of optimal sensor placement algorithms via implementation on an innovative timber structure. J. Bakker, D.M. Frangopol, K. van Breugel eds. Life-Cycle of Engineering Systems: Emphasis on Sustainable Civil Infrastructure. Proceedings of the Fifth International Symposium on Life-Cycle Civil Engineering (IALCCE 2016), Delft, The Netherlands, October 16-19, 2016, 260267.

[11] C. Papadimitriou, Optimal sensor placement methodology for parametric identification of structural systems, Journal of Sound and Vibration, 278, 923-947, 2004.

[12] J-H. Han, I. Lee, Optimal placement of piezoelectric sensors and actuators for vibration control of a composite plate using genetic algorithms, Smart Materials and Structures, $\mathbf{8}$, 257-267, 1999.

[13] K. Worden, A.P. Burrows, Optimal sensor placement for fault detection, Engineering Structures, 23, 885-901, 2001.

[14] D.C. Kammer, Sensor placement for on-orbit modal identification and correlation of large space structures, Journal of Guidance, Control, and Dynamics, 14, 251-259, 1991.

[15] D.C. Kammer, Effects of noise on sensor placement for on-orbit modal identification of large space structures, Journal of dynamic systems, measurements and controlTransactions of the ASCE, 114, 436-443, 1992.

[16] C. Papadimitriou, G. Lombaert, The effect of prediction error correlation on optimal sensor placement in structural dynamics, Mechanical Systems and Signal Processing, 28, 105-127, 2012. 
[17] B. Lin, B. Recke, J.K.H. Knudsen, S.B. Jørgensen, A systematic approach for soft sensor development, Computers and Chemical Engineering, 31, 419-425, 2007.

[18] L. Liu, S.M. Kuo, M. Zhou, Virtual sensing techniques and their applications, Proceedings of the 2009 IEEE International Conference on Networking, Sensing and Control, Okayama, Japan, March 26-29, 2009, 31-36.

[19] J. Kullaa, Robust damage detection in the time domain using Bayesian virtual sensing with noise reduction and environmental effect elimination capabilities, Journal of Sound and Vibration, 473, 115232, 2020.

[20] J. Kullaa, Sensor validation using minimum mean square error estimation, Mechanical Systems and Signal Processing, 24, 1444-1457, 2010.

[21] J. Kullaa, Bayesian virtual sensing in structural dynamics, Mechanical Systems and Signal Processing, 115, 497-513, 2018.

[22] H. Sohn, Effects of environmental and operational variability on structural health monitoring, Philosophical Transactions of the Royal Society A: Mathematical, Physical and Engineering Sciences, 365, 539-560, 2007.

[23] L.L. Scharf, Statistical signal processing: detection, estimation, and time series analysis. Addison-Wesley, 1991.

[24] J. Kullaa, Optimal sensor placement of Bayesian virtual sensors. W. Desmet, B. Pluymers, D. Moens, S. Vandemaele eds. Proceedings of ISMA2020, International Conference on Noise and Vibration Engineering, Leuven, Belgium, September 7-9, 2020, 973-985.

[25] H. Stark, J.W. Woods, Probability and random processes with applications to signal processing. $3^{\text {rd }}$ edition. Prentice-Hall, 2002.

[26] J. Kullaa, Whitening transformation in damage detection. A.E. Del Grosso, P. Basso eds. Smart structures: Proceedings of the 5th European Conference on Structural Control EACS 2012, Genoa, Italy, June 18-20, 2012.

[27] K. Worden, D. Allen, H. Sohn, C.R. Farrar, Damage detection in mechanical structures using extreme value statistics, SPIE Proceedings, Vol. 4693, 9th Annual International Symposium on Smart Structures and Materials, San Diego, CA, 2002, 289-299.

[28] D.C. Montgomery, Introduction to statistical quality control, 3rd edition, Wiley, 1997. 